\title{
15 Unsaturated fatty acids
}

Fatty acids are long-chain hydrocarbons. They are called unsaturated fatty acids when they lack the maximum possible number of hydrogen atoms attached to every carbon atom. For example, if stearic acid, which has 18 carbon atoms and frequently occurs in animal and plant fats, is missing 2 hydrogen atoms, then a double bond is present and the resulting acid is oleic acid. Its polyunsaturated derivatives linoleic acid (omega-6) and linolenic acid (omega-3) have 2 and 3 double bonds, respectively. Omega- 3 and omega- 6 fatty acids are both named for the position of the first double bond in the carbon chain.

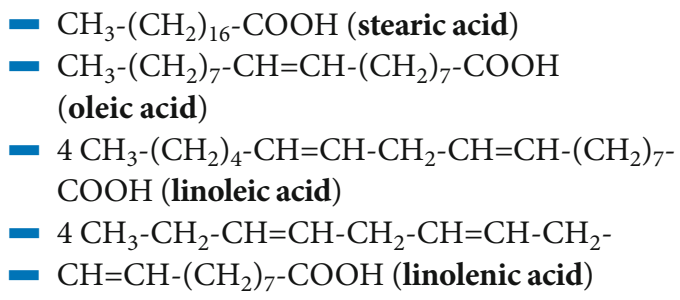

Our bodies must obtain these two polyunsaturated acids, also called essential fatty acids, from our food. Linoleic acid is found in grains, soybeans and vegetable oils. Linolenic acid is found in leafy green vegetables and in vegetable oils. Eicosapentaenoic acid (20 carbon atoms, 5 double bonds) and docosahexaenoic acid ( 22 carbon atoms, 6 double bonds) are even longer-chain unsaturated omega- 3 fatty acids. They are mainly found in fatty marine fish and can be produced from linoleic acid to a limited extent by the human body.
According to data from large-scale studies, omega-3 fatty acids reduce the risk of age-related macular degeneration (AMD) (Chong et al. 2009, Christen et al. 2011), but do not reduce the risk of further progression to advanced AMD in persons with pre-existing conditions (Chew 2013). Furthermore, findings suggest that omega- 3 fatty acids may protect against cellular aging (Farzaneh-Far et al. 2010). It is known that abundant fish consumption naturally slows down the shortening of leukocyte telomeres ( $\downarrow$ Chapter 47 ).

One important effect of polyunsaturated omega-3 fatty acids, which are part of any varied diet, is protection against the fatal complications of coronary heart disease (Roncaglioni et al. 2013). Furthermore, the various unsaturated fatty acids are the basic building blocks for the production of prostaglandins (tissue hormones) that affect both vessel size and inflammatory processes. These hormones also promote the formation of leukotrienes, which have an inflammatory and hyperalgesic (painenhancing) effect. Finally, they play an important role in thromboxane synthesis within platelets. Thromboxane promotes platelet aggregation and clotting in response to injuries. Under unfavorable circumstances, however, it promotes thrombosis as well. One physiologic antagonist of thromboxane is prostacyclin, a prostaglandin synthesized by endothelial cells $(\checkmark$ Chapter 51 ). For optimal synthesis of these cell messengers, the ratio of omega- 6 to omega-3 fatty acids in our food should be approximately 5 to $\mathbf{1}$. In Germans, for instance, this ratio is unfortunately around 20 to 1 . 